\title{
Downregulated miR-302d-3p promotes chondrocyte proliferation and migration by regulation of Unc-51-like kinase 1
}

\author{
SHANGZENG WANG ${ }^{1}$, YONGZHI ZHENG ${ }^{1}$, ZHENG HU ${ }^{2}$, ZHEN WANG $^{3}$, YINGJIE ZHANG ${ }^{3}$ and LICHENG WEI $^{2}$ \\ ${ }^{1}$ Department of Orthopedics, The Second Affiliated Hospital of Henan University of Traditional Chinese Medicine, \\ Zhengzhou, Henan 450002; ${ }^{2}$ Department of Orthopedics, Changsha Hospital of Traditional Chinese Medicine, \\ Changsha Eighth Hospital, Changsha, Hunan $410100 ;{ }^{3}$ Graduate School of Henan University of \\ Traditional Chinese Medicine, Zhengzhou, Henan 450003, P.R. China
}

Received December 7, 2018; Accepted June 19, 2019

DOI: $10.3892 /$ ijmm.2019.4267

\begin{abstract}
Osteoarthritis (OA) is a common musculoskeletal disease and is related to the function of chondrocytes. The aim of the present study was to investigate the effects of miR-302d-3p on chondrocytes. Quantitative PCR (qPCR) was conducted to detect mRNA expression, while western blotting was performed to investigate protein expression in these cells. RNAs mimics, inhibitors and small interfering (si)RNAs were respectively transfected into chondrocytes (CHON-001 cell line), after which, a Cell Counting Kit-8 assay was performed to detect chondrocyte viability. Giemsa staining of the cells was also conducted to analyze the colony formation ability of the cells. Additionally, cell apoptosis was evaluated with an apoptosis detection kit using flow cytometry. A scratch-wound assay was conducted to investigate cell migration. Bioinformatics analysis using TargetScan 7.2 revealed the potential the target gene of microRNA (miR)-302d-3p; a dual luciferase reporter assay determined the target gene. Suppression of miR-302d-3p increased the viability of cells, cell colony number and migration; CHON-001 cell apoptosis was also inhibited. miR-302d-3p mimics decreased the luciferase activity of reporter plasmids containing the wildtype 3'-untranslated region of Unc-51-like kinase 1 (ULK1). siULK1 decreased CHON-001 cell viability and migration. Furthermore, siULK1 promoted the expression of phosphorylated $\mathrm{I \kappa B} \alpha$ and $\mathrm{p} 65$, while miR-302d-3p inhibitor suppressed
\end{abstract}

Correspondence to: Dr Licheng Wei, Department of Orthopedics, Changsha Hospital of Traditional Chinese Medicine, Changsha Eighth Hospital, 22 Xingsha Avenue, Changsha, Hunan 410100, P.R. China

E-mail: lichengw_weilc@163.com

Abbreviations: ULK1, Unc-51-like kinase 1; OA, osteoarthritis; RT-qPCR, reverse transcription-quantitative polymerase chain reaction; miRNAs, microRNAs; STK, serine/threonine protein kinase

Key words: microRNA-302d-3p, CHON-001, osteoarthritis, ULK1, inflammation the expression of phosphorylated I $\mathrm{B} \alpha$ and $\mathrm{p} 65$. Inhibition of miR-302d-3p could promote the proliferation and migration, and inhibit the apoptosis of chondrocytes, potentially by upregulating ULK1; thus, inflammation may be suppressed. The findings of the present study suggest miR-302d-3p and ULK1 as potential therapeutic targets for the prevention and treatment of OA.

\section{Introduction}

At present, osteoarthritis (OA) is one of the most common chronic and musculoskeletal diseases, and lowers patient quality of life (1). In the United States of America, over 26 million adults have $\mathrm{OA}$ and $\sim 5.6$ million cases possess lower extremity $\mathrm{OA} ; \sim 13$ million people $>60$ years old have radiographic OA in this region of the world (2). Muthuri et al (3) reported that those with joint injury have a higher risk of developing OA than those with no history of joint injury (4). Additionally, genetic factors contribute to the development of $\mathrm{OA}$; the heritability of OA is $>50 \%$ (5). Other factors, including obesity, age, female gender and bone mineral density, increase the risk of OA development (6).

$\mathrm{OA}$ is characterized by stiffness, joint pain and limitation of joint movement, and occasional effusion and local inflammation (7). Constant pain frequently occurs in patients with late OA stage (8). Pain causes changes to the bone structure in affected joints and disrupts the balance in mechanisms underlying structural change, peripheral and central pain sensitivity (9). OA patients frequently exhibit higher levels of inflammatory cytokines, such as interleukin (IL)-1 $\beta$ and tumor necrosis factor (TNF)- $\alpha$ (10).

MicroRNAs (miRNAs/miRs) are large family of noncoding RNA molecules ( 22 nucleotides) and are linked with biological processes, including cellular apoptosis, proliferation and differentiation $(11,12)$. In addition, miRNAs bind to the 3'-untranslated region (3'-UTR) of target messenger RNA (mRNA), in which miRNAs can block the translation of mRNA and promote the degradation of mRNA (11). miRNAs can regulate genes expression and signaling pathways in human conditions, such as neuropathic pain (13). The miR-302/367 cluster is located in the intron of the $4 q 25$ region of chromosome 4 and is transcribed by RNA polymerase II; 
the miR-302/367 cluster comprises five members, including miR-367, -302a, -302b, $-302 \mathrm{c}$ and $-302 \mathrm{~d}$ (14). It has been reported that the miR-302/367 cluster serves an important role in regulating the G1-S transition of the cell cycle $(14,15)$. Furthermore, recent studies supported that the miR-302/367 cluster attenuated or negatively regulated inflammation $(16,17)$.

To the best of our knowledge, the present study revealed that the cartilaginous tissue of OA patients exhibited higher miR-302d-3p expression levels compared with in normal cartilaginous tissue. Therefore, we hypothesized that inhibition of miR-302d-3p may prevent the progression of OA by promoting proliferation and healing, and inhibiting inflammation in chondrocytes in vivo.

\section{Materials and methods}

Reverse transcription-quantitative polymerase chain reaction (RT-qPCR). From March 2017 to March 2018, a total of 32 cartilaginous tissues each from patients without osteoarthritis (OA) and with OA were obtained for analysis; in addition, 6 groups of treated-CHON-001 cells (American Type Culture and Collection) were acquired. The cells were treated with miR-302d-3p mimics, miR-302d-3p mimics control, miR-302d-3p inhibitor, miR-302d-3p inhibitor control, respectively. All patients provided written informed consent form before the acquisition of samples. The present study was approved by the ethics committees and health authorities of The Second Affiliated Hospital of Henan University of Traditional Chinese Medicine (approval. no. R201703050089). The patients were aged between 18-60 years and were male. Patients were included in the trial if they met clinical criteria for OA according to The American College of Rheumatology (18). All patients with joint deformities, rheumatoid arthritis, septic arthritis, ankylosing spondylitis, gout, hematopoietic system, or other serious diseases were excluded. Tissues samples were cut into pieces and RNA was extracted using TRIzol ${ }^{\circledR}$ (Thermo Fisher Scientific, Inc.) and a purification kit (Thermo Fisher Scientific, Inc.). Total RNA was extracted from CHON-001 cells using TRIzol ${ }^{\circledR}$ reagent (Thermo Fisher Scientific, Inc.). cDNAs were synthesized by cDNA kit (Invitrogen; Thermo Fisher Scientific, Inc.) according to the manufacturer's protocols. Subsequently, qPCR experiments were performed with the SYBR Premix Ex Taq ${ }^{\mathrm{TM}}$ Real-Time PCR kit (Takara Bio, Inc., Otsu, Japan). qPCR thermocycling conditions were: Initial denaturation at $95^{\circ} \mathrm{C}$ for $1 \mathrm{~min}$, followed by 50 cycle of $95^{\circ} \mathrm{C}$ for $30 \mathrm{sec}, 55^{\circ} \mathrm{C}$ for $45 \mathrm{sec}$ and $72^{\circ} \mathrm{C}$ for $35 \mathrm{sec}$.

The sequence of primers employed for qPCR were synthesized by Sangon Biotech Co., Ltd. (Table I). Data from qPCR were analyzed with the $2^{-\Delta \Delta \mathrm{Cq}}$ method (19). miR-302d-3p or Unc-51-like kinase 1 (ULK1) mRNA expression from the tissue of one patient tissue (without OA) was selected as the control. U6 and GAPDH were used as internal controls.

Cell culture and transfection. CHON-001 cells are derived from human cartilage and were obtained from the American Type Culture and Collection; the cell line was used to investigate chondrocyte function in the present study (20). CHON-001, the human chondrocyte cell line, was cultured in
Table I. Sequences of primers employed for reverse transcription-quantitative polymerase chain reaction.

\begin{tabular}{ll}
\hline Primer & \multicolumn{1}{c}{ Sequence $\left(5^{\prime}-3^{\prime}\right)$} \\
\hline $\begin{array}{l}\text { miR-302d-3p } \\
\text { U6 }\end{array}$ & F: GCGTAAGTGCTTCCATGTTTGTGTGT \\
& F: CGGGTTTGTTTTGCATTTCT \\
RLK1 & F: AGTCCCAGCATGAACAGCTT \\
& R: CCAGAGCAACATGATGG \\
GAPDH & F: CAGCCTCAAGATCATCAGCA \\
& R: TGTGGTCATGAGTCCTTCCA
\end{tabular}

miR, microRNA; F, forward; R, reverse; ULK1, Unc-51-like kinase 1.

Table II. Primary antibodies employed for western blotting.

\begin{tabular}{|c|c|c|}
\hline Antibody & Cat. no. & Target protein weight $(\mathrm{kDa})$ \\
\hline ULK1 & 8,054 & 150 \\
\hline $\mathrm{I} \kappa \mathrm{B} \alpha$ & 4,814 & 39 \\
\hline $\mathrm{p}-\mathrm{I} \kappa \mathrm{B} \alpha$ & 9,246 & 40 \\
\hline p65 & 8,242 & 65 \\
\hline p-p65 & 3,039 & 65 \\
\hline GAPDH & 4,292 & 37 \\
\hline
\end{tabular}

p, phosphorylated; ULK1, Unc-51-like kinase 1.

high-glucose Dulbecco's modified Eagle's medium (DMEM; Gibco; Thermo Fisher Scientific, Inc.) supplemented with $0.1 \mathrm{mg} / \mathrm{ml} \mathrm{G}-418$ (Gibco; Thermo Fisher Scientific, Inc.) and $10 \%$ fetal bovine serum (FBS; Gibco; Thermo Fisher Scientific, Inc.) at $37^{\circ} \mathrm{C}$ at $5 \% \mathrm{CO}_{2}$ in an incubator (Thermo Fisher Scientific, Inc.). The culture medium was denoted as complete medium.

CHON-001 cells were seeded in a 6-well plate (Corning Inc.), and miR-302d-3p mimics or inhibitors mixed with Lipofectamine $^{\circledR}$ (Invitrogen; Thermo Fisher Scientific, Inc.) and DMEM; cells were incubated cells for $3 \mathrm{~h}$ at $37^{\circ} \mathrm{C}$. Complete medium was added to cultured cells for $48 \mathrm{~h}$ at $37^{\circ} \mathrm{C}$ after $3 \mathrm{~h}$ incubation. The concentration of the mimics, inhibitors and siRNAs were $50 \mathrm{nM}$. The transfection reagent was used in accordance with the manufacturer's protocols. miR-302d-3p mimics, miR-302d-3p mimics control, miR-302d-3p inhibitor, miR-302d-3p inhibitor control, small interfering RNA (si)-negative control (NC) and siULK1 were synthesized by Sigma-Aldrich (Merck KGaA). The sequences of siRNA, mimics and inhibitors used in the present study were as follows: siULK1: 5'-GCACAGAGACCGTGGG CAA-3'; miR-302d-3p mimics: 5'-UAAGUGCUUCCAUGU UUGAGUGU-3'; miR-302d-3p inhibitor: 5'-ACACUCAAA CAUGGAAGCACUUA-3'; NC-mimics: 5'-UUCUCCGAA CGUGUCACGUTT-3'; NC-inhibitor: 5'-CAGUACUUUUGU GUAGUACAA-3'. Following transfection for $48 \mathrm{~h}$, the cells were harvested and used for the subsequent experiments. Nontransfected cells were used as blank controls. 
A
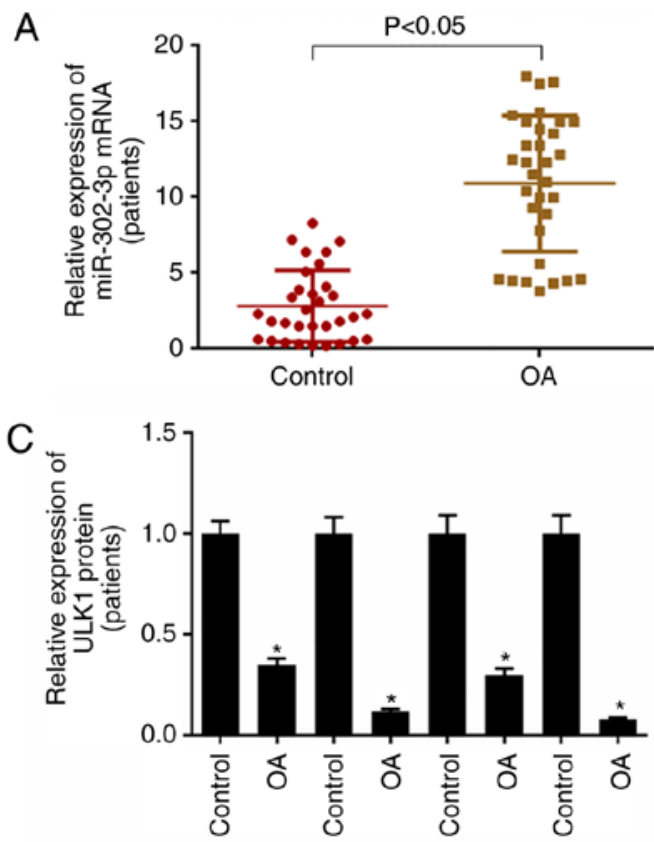

B
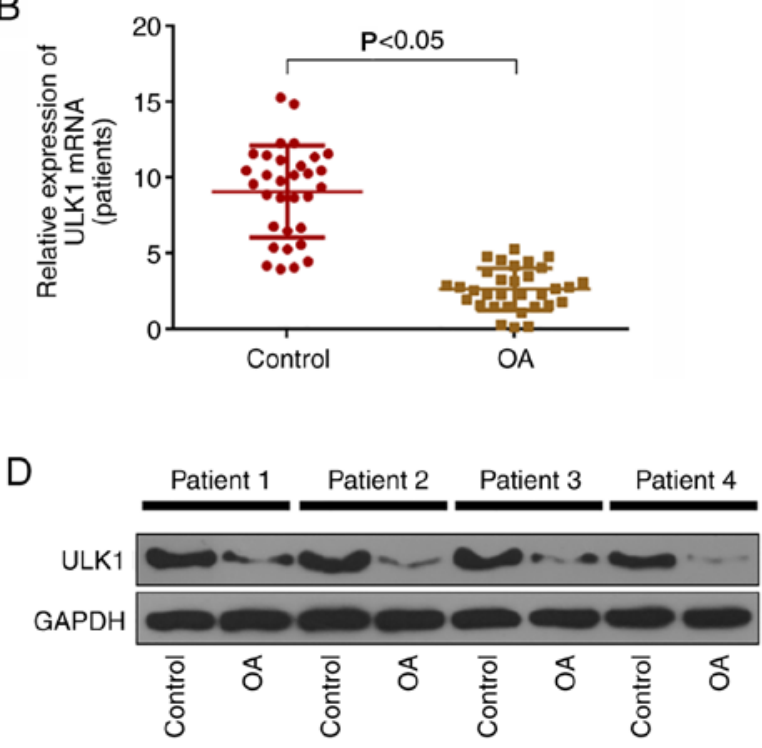
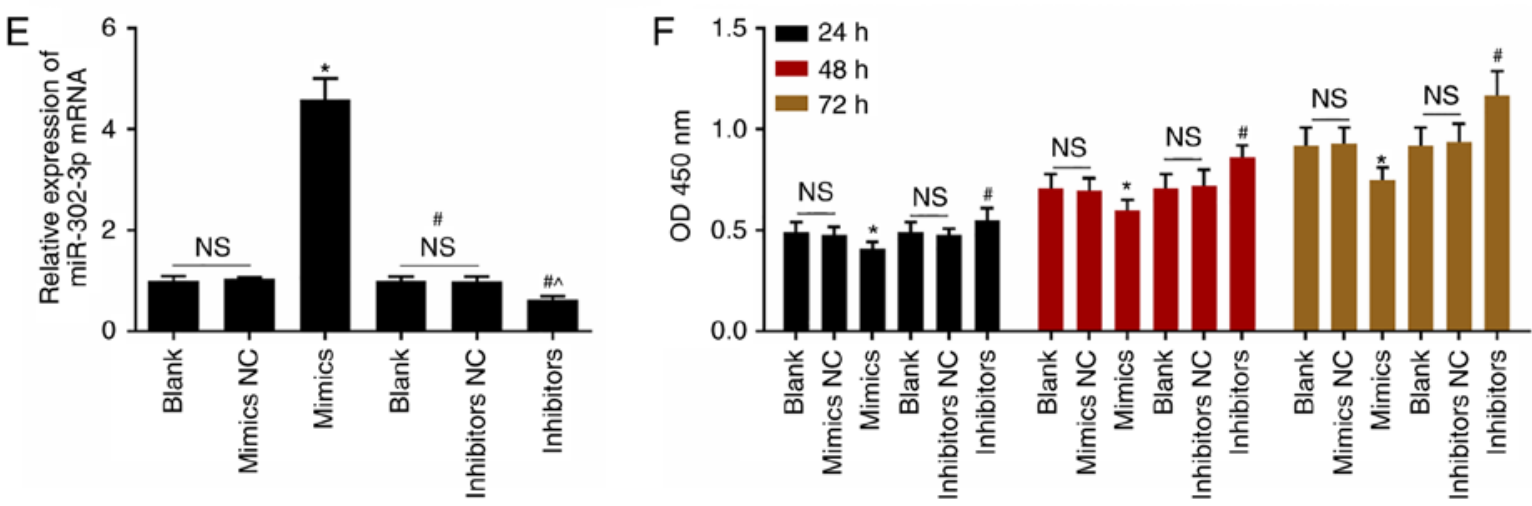

Figure 1. Expression of miR-302d-3p and ULK1 in patient samples, and the effects of miR-302d-3p inhibitor or mimics on CHON-001 cell proliferation. RT-qPCR the expression of (A) miR-302d-3p mRNA and (B) ULK1 mRNA in cartilaginous tissue of patients with or without OA. (C and D) Western blotting for the detection of ULK1 protein expression in patients with or without OA. (E) RT-qPCR was conducted to detect miR-302d-3p expression in CHON-001 cells after transfection with miR-302d-3p, inhibitors or NC for 48 h. (F) A Cell Counting Kit-8 assay was performed to determine CHON-001 cell viability at the wavelength of $450 \mathrm{~nm}$. The values were presented as the mean \pm standard deviation; data were analyzed via one way ANOVA. "P<0.05 vs. Blank group; ${ }^{*} \mathrm{P}<0.05$ vs. mimics group and ${ }^{\wedge} \mathrm{P}<0.05$ vs. inhibitors $\mathrm{NC}$ group. miR, microRNA; NC, negative control; OA, osteoarthritis; OD, optical density; RT-qPCR, reverse transcription-quantitative polymerase chain reaction; ULK1, Unc-51-like kinase 1.

To further investigate the mechanism of miR-302d-3p on the proliferation and migration of chondrocytes, the cells were divided into 6 groups: Blank group (nontransfected cells), siNC group (cells were transfected with siNC); siULK1 group (cells were transfected with siULK1); inhibitors group (cells were transfected with miR-302d-3p inhibitors); inhibitors + siNC (cells were transfected with miR-302d-3p inhibitors and siNC) and inhibitors + siULK1 group (cells were transfected with miR-302d-3p inhibitors and siULK1).

Cell viability. CHON-001 cells $\left(2 \times 10^{3}\right.$ cells/well $)$ were seeded into a 96-well plate (Corning Inc.) for 24,48 and $72 \mathrm{~h}$ following transfection. Cell Counting Kit-8 solution (CCK-8; Sigma-Aldrich; Merck KGaA) was diluted with DMEM (Gibco; Thermo Fisher Scientific, Inc.) (1:9). $100 \mu 1$ CCK-8 mixture was added to cells, which were incubated for $1 \mathrm{~h}$ after the medium was discarded. The absorbance was detected at a wavelength of $450 \mathrm{~nm}$ using a Multiskan spectrophotometer.
Colony formation assay. A colony formation assay was performed to evaluate cell proliferation (21). $\mathrm{CHON}-001$ cells were digested and resuspended into single cell suspension using $0.25 \%$ trypsin-EDTA (Gibco; Thermo Fisher Scientific, Inc.) and complete medium after the cells were treated as aforementioned. The cells were seeded into $35 \mathrm{~mm}$ culture dishes (Corning Inc.; 200 purified cells in each dish) and incubated at $37^{\circ} \mathrm{C}$ in a $5 \% \mathrm{CO}_{2}$. incubator (Thermo Fisher Scientific, Inc.), G418 (700 $\mu \mathrm{g} / \mathrm{ml}$; Abcam) was added to the medium and mixed to detect positive cell clones for 14 days until visible cell clones emerged. Fresh medium was replaced every 3 days. PBS was used to wash the cells three times after the complete medium was discarded. The cells were fixed with $4 \%$ paraformaldehyde (Beijing Solarbio Science \& Technology, Co. Ltd.) for $15 \mathrm{~min}$ at room temperature and stained with Giemsa (Beijing Solarbio Science \& Technology, Co. Ltd.) for 10-30 min at room temperature. Then, the cells were washed with PBS three times again, after which the number of cell colonies was calculated. 
A
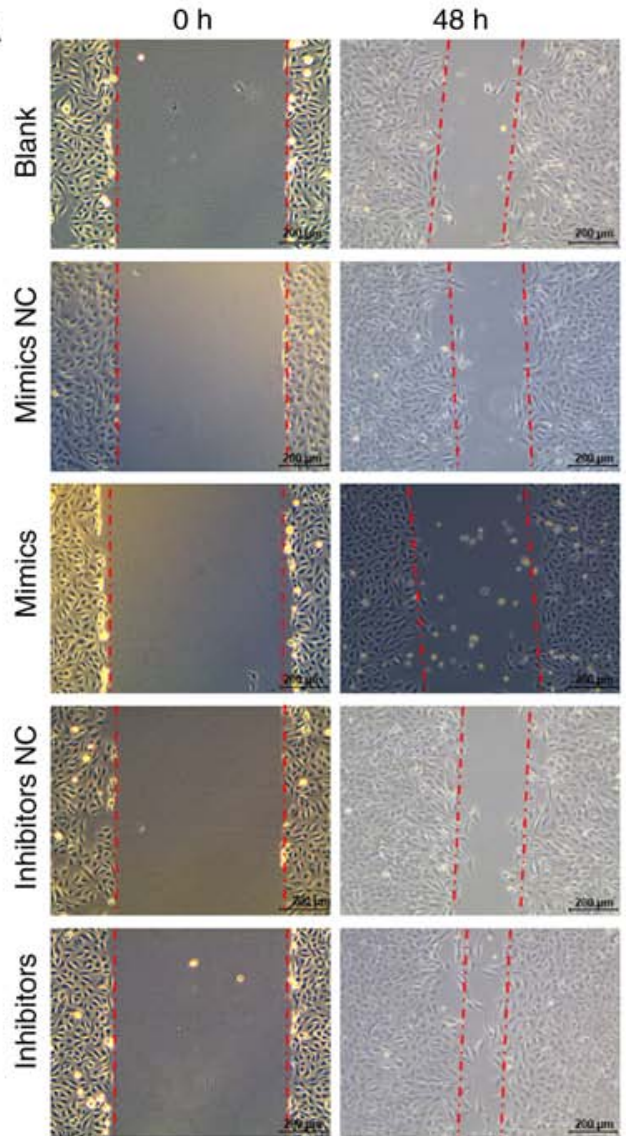

$48 \mathrm{~h}$
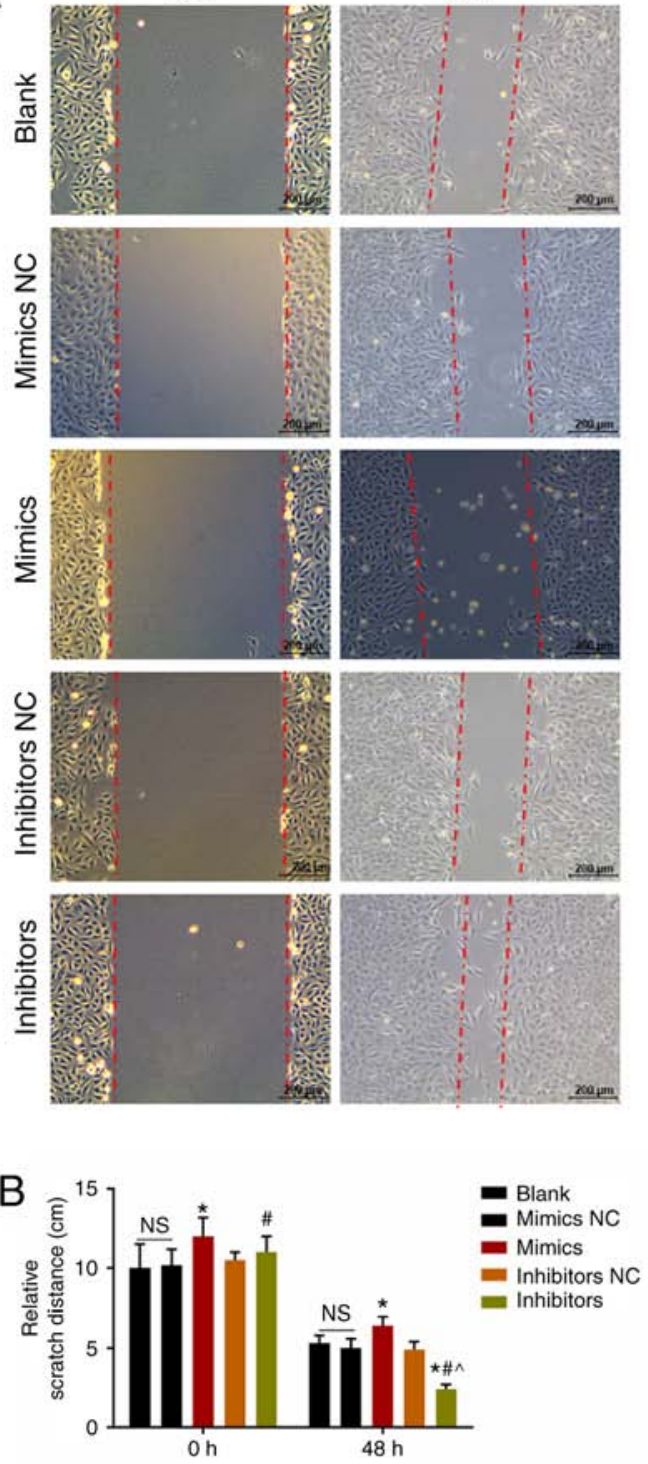

C
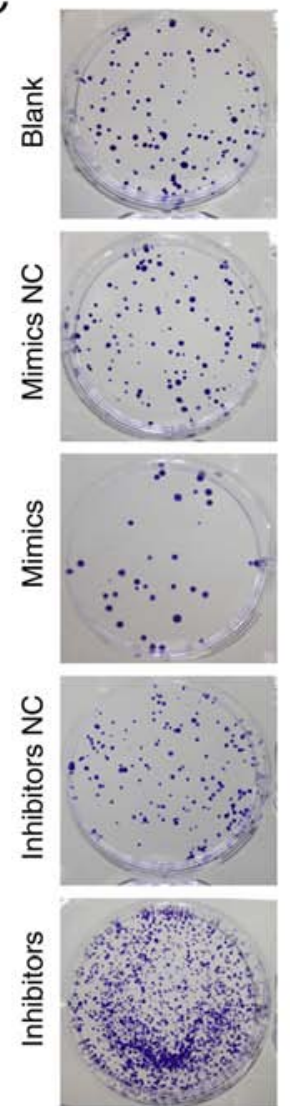

$\mathrm{E}$
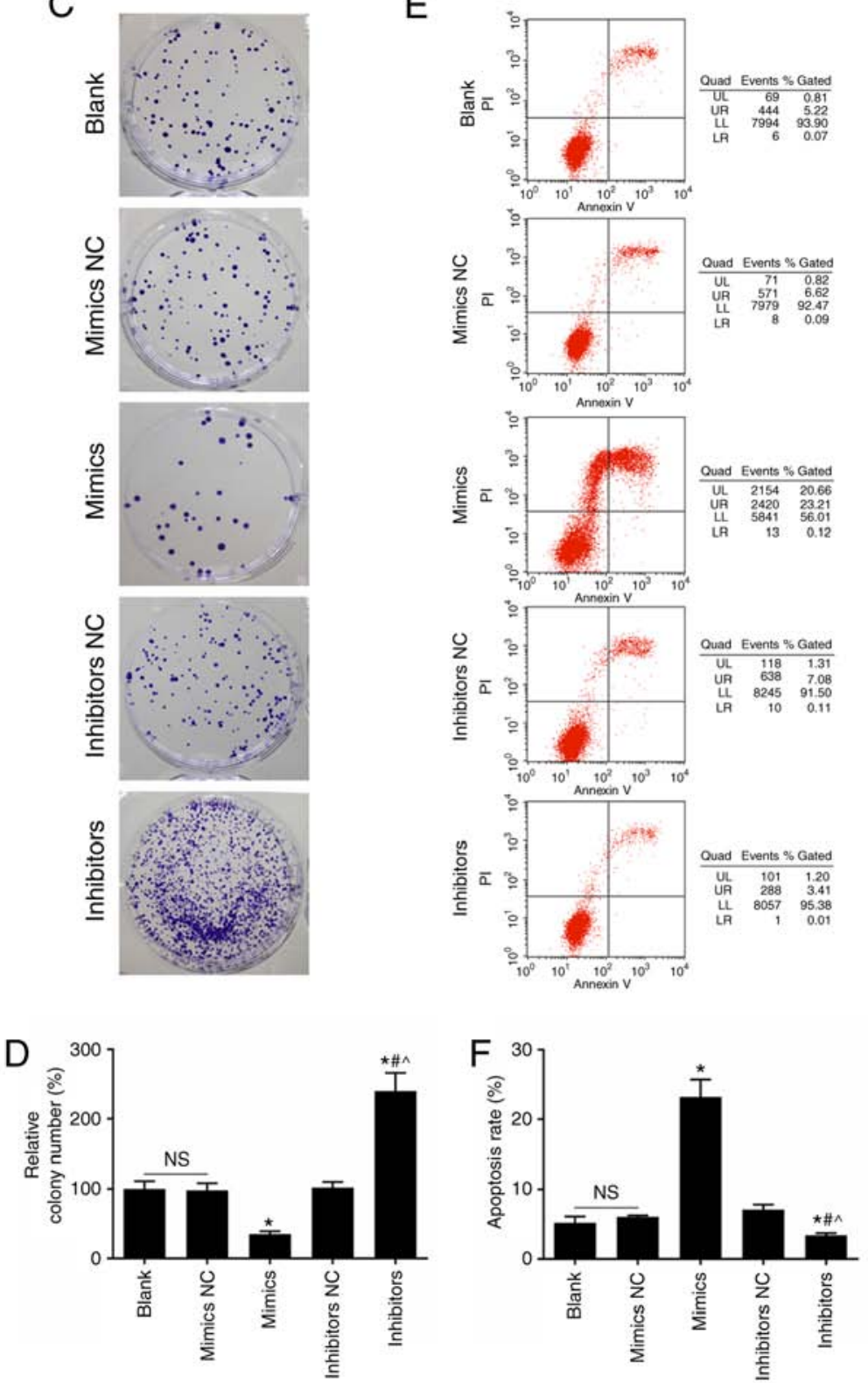

Figure 2. Effects of miR-302d-3p inhibitor and mimics on CHON-001 cell migration, colony number and apoptosis. (A and B) CHON-001 cells were seeded on a $35-\mathrm{mm}$ plate $48 \mathrm{~h}$ following transfection. The scratch-wound assay revealed the effects of transfection on the migration of CHON-001 cells. Scale bar, $200 \mu \mathrm{m}$. (C and D) Giemsa-stained cell colonies. (E and F) An Apoptosis detection kit was used to detect CHON-001 cell apoptosis by flow cytometry. The values were presented as the mean \pm standard deviation; data were analyzed by one way ANOVA. ${ }^{*} \mathrm{P}<0.05$ vs. Blank group; ${ }^{\text {"}} \mathrm{P}<0.05$ vs. mimics group and ${ }^{\wedge} \mathrm{P}<0.05$ vs. inhibitors NC group. NS, not significant; miR, microRNA; NC, negative control.

Apoptosis analysis. CHON-001 cells were digested with $0.25 \%$ trypsin-EDTA and resuspended in FBS-free DMEM after cells were treated as aforementioned for $48 \mathrm{~h}$. An apoptosis detection kit with Annexin V-fluorescein isothiocyanate and propidium iodide (Invitrogen; Thermo Fisher Scientific, Inc.) was employed according to the manufacturer's protocols. The BD FACSCanto flow cytometer (BD Biosciences) was used to analyze apoptotic cells, analysis of data was performed using the FACSDiva software version 6.1.2 (BD Biosciences).

Scratch-wound assay. A scratch-wound assay can be applied to evaluate cell migration (22). CHON-001 cells were resuspended in complete medium with $1 \%$ FBS (Gibco; Thermo Fisher Scientific, Inc.) after the cells were treated as aforementioned for $48 \mathrm{~h}$. Then, the cells were seeded in a $35-\mathrm{mm}$ plate (Corning Inc.) at a density of $5 \times 10^{3}$ cells $/ \mathrm{cm}^{2}$. A sterile $200 \mu \mathrm{l}$ pipette tips (Sigma-Aldrich; Merck KGaA) were used to scratch the cell monolayer, and the cells were washed with PBS. The media was replaced with complete medium containing $1 \%$ FBS once every $12 \mathrm{~h}$. After $48 \mathrm{~h}$, the scratches were observed under a light microscope (Olympus Corporation; magnification, x100). The distances are different between groups at time $0 \mathrm{~h}$ were calculated.

Dual-luciferase reporter assay. TargetScan7.2 (http://www. targetscan.org/vert_72/) was used to predict the target gene of miR-302d-3p, and the dual luciferase reporter assay was used to confirm the findings. The wild-type (wt) or mutant 
A

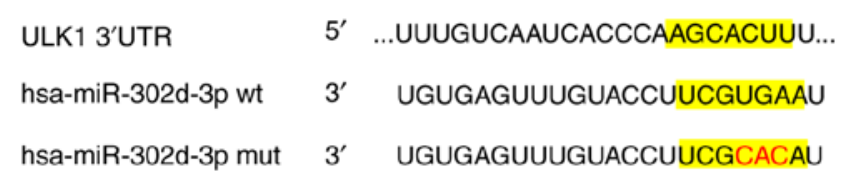

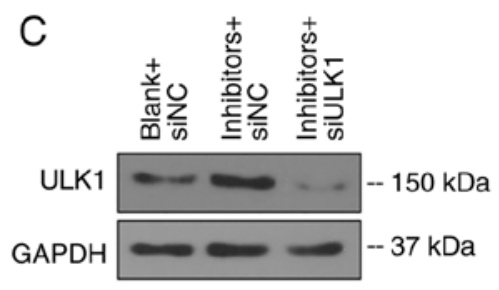

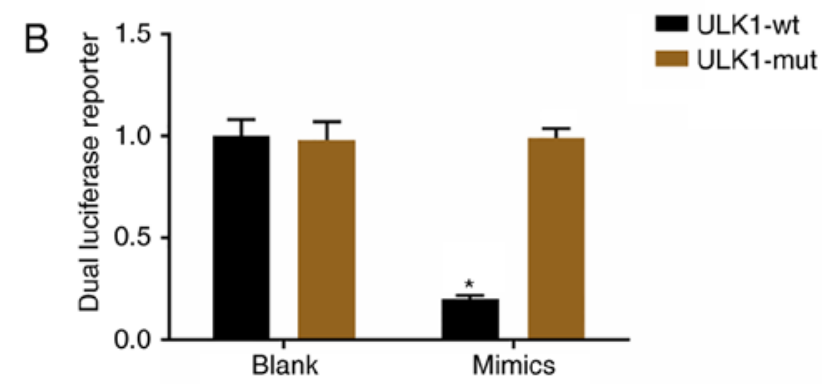

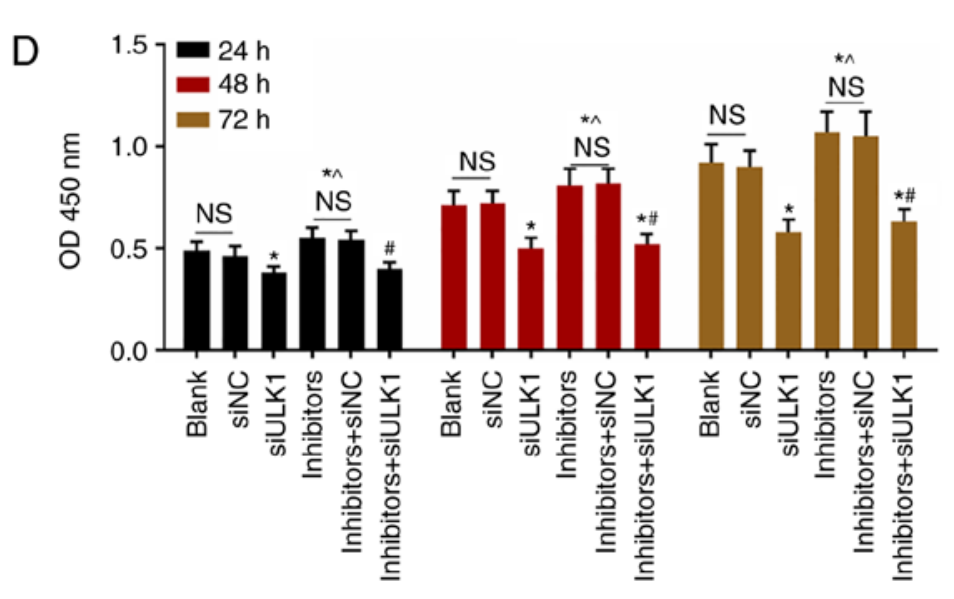

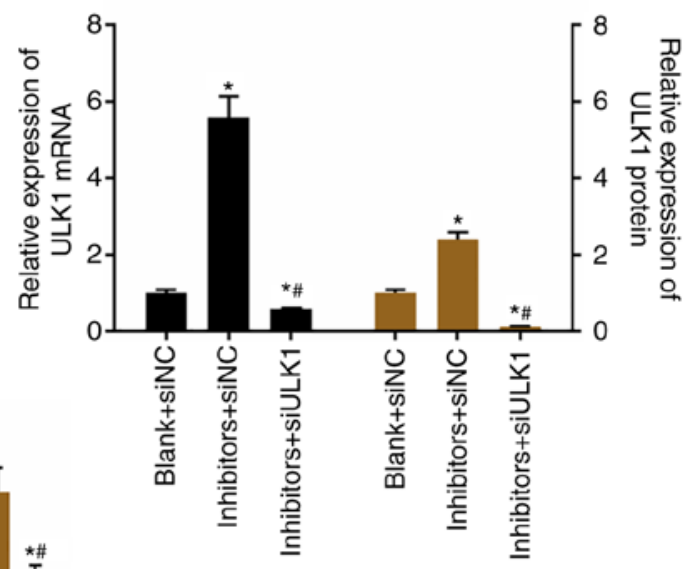

Figure 3. siULK1 inhibits CHON-001 cell viability. (A) TargetScan7.2 analysis revealed ULK1 as a target gene of miR-302d-3p. (B) Dual luciferase report assay was conducted to determine the binding of miR-302d-3p to ULK1. (C) Reverse transcription-quantitative polymerase chain reaction and western blotting indicated the effects of miR-302d-3p inhibitor on ULK1 expression. (D) Cell Counting Kit-8 assay demonstrated that siULK1 inhibited CHON-001 cell viability. The values were presented as the mean \pm standard deviation; data were analyzed by one way ANOVA. ${ }^{*} \mathrm{P}<0.05$ vs. Blank group; ${ }^{\#} \mathrm{P}<0.05$ vs. inhibitor + siNC group and ${ }^{\wedge} \mathrm{P}<0.05$ vs. siULK1 group. Hsa, homo sapiens; miR, microRNA; Mut, mutant; NC, negative control; si, small interfering RNA; ULK1, Unc-51-like kinase 1; wt, wild-type.

(mut) ULK1 3'-UTR was cloned into psi-CHECK-2 (Promega Corporation) according to the manufacturer's instructions. Cells were transfected with miR-302d-3p mimics or miRNA control for $48 \mathrm{~h}$. The Luciferase assay reagent II (100 $\mu \mathrm{l})$ and 1X Stop\&Glo ${ }^{\circledR}$ reagent (100 $\mu \mathrm{l}$; Promega Corporation) were added to the cells, and luciferase activities were detected using the GloMax ${ }^{\circledR}$ Discover Multimode Microplate Reader (cat. no. GM3000; Promega Corporation) according to the manufacturer's instructions. Luciferase activity was normalized to Renilla luciferase activity.

Western blotting. Total protein was extracted from CHON-001 cells and tissues with cell extraction kit (Thermo Scientific, Waltham, MA, USA) by centrifuging samples at $4^{\circ} \mathrm{C}, 6,000 \mathrm{x}$ g for $10 \mathrm{~min}$. A BCA kit was used to determine the concentration of extracted protein. Total protein $(20 \mu \mathrm{g})$ and a pre-stained protein ladder (Thermo Fisher Scientific, Inc.) were separated by $10 \%$ SDS-PAGE and transferred to polyvinylidene difluoride membranes (Sigma-Aldrich; Merck KGaA). Membranes were stained with $1 \mathrm{X}$ ponceau-S (Beijing Solarbio Science \& Technology, Co. Ltd.) following transfer to ensure consistent loading of total protein per lane. The protein membranes were blocked with $5 \%$ bovine serum albumin (Sigma-Aldrich; Merck KGaA) at room temperature. Primary antibodies (Table II; Cell Signaling Technologies, Inc.) were applied and membranes were incubated at $4^{\circ} \mathrm{C}$ overnight. Secondary antibodies (cat. nos. 7074 and 7076; Cell Signaling Technologies, Inc.) were applied for $2 \mathrm{~h}$ at room temperature. Primary and secondary antibodies were diluted with TBST with Tween-20, as specified by the supplier. An ECL kit (Sigma-Aldrich; Merck KGaA) was employed to visualize proteins. Stains were developed with X-ray film (Fuji, Tokyo, Japan). The densitometry was performed using the Bio-Rad ChemiDoc system with Image Lab software version 6.0 (Bio-Rad Laboratories, Inc., Hercules, CA, USA).

Statistical analysis. The aforementioned experiments were independently repeated at least three times. The results were presented as the mean \pm standard deviation. Analysis was conducted with one way ANOVA using SPSS 21.0 (IBM Corp.) followed by Tukey's Honest Significant Difference post hoc test. $\mathrm{P}<0.05$ was considered to indicate a statistically significant difference. 
A
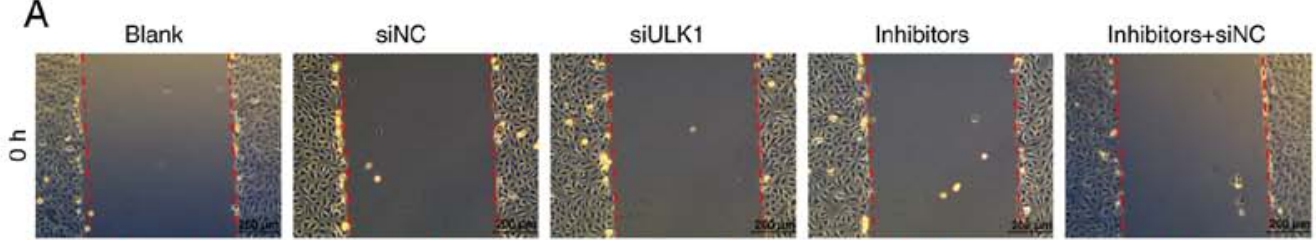

Inhibitors+siULK1
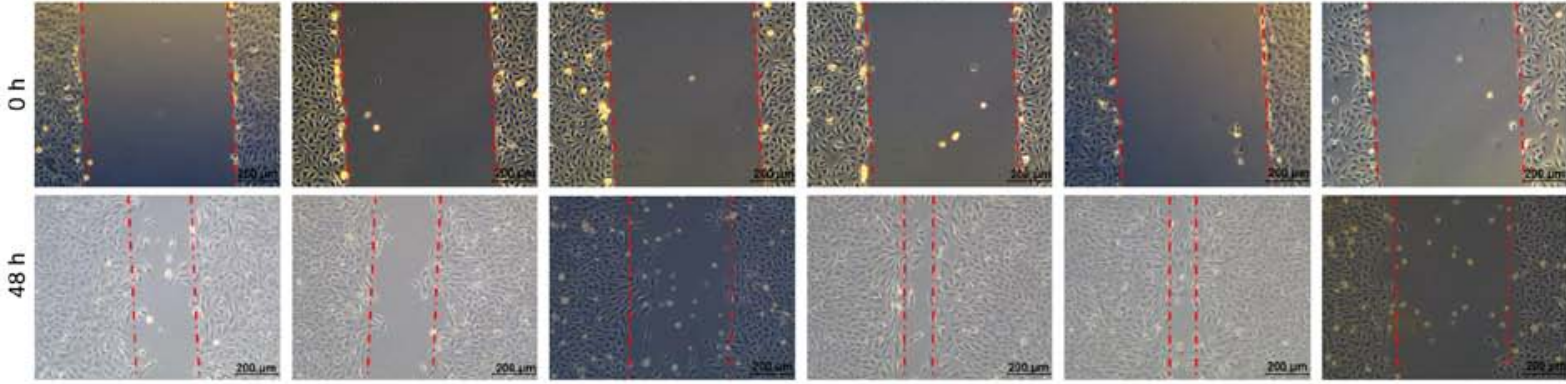

B

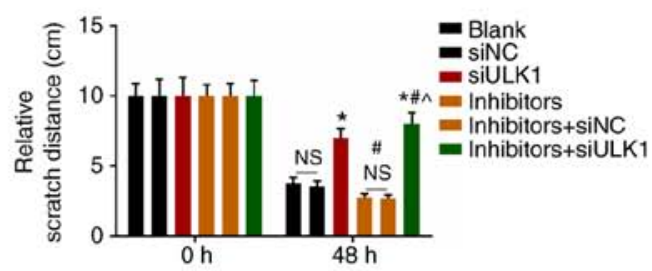

C
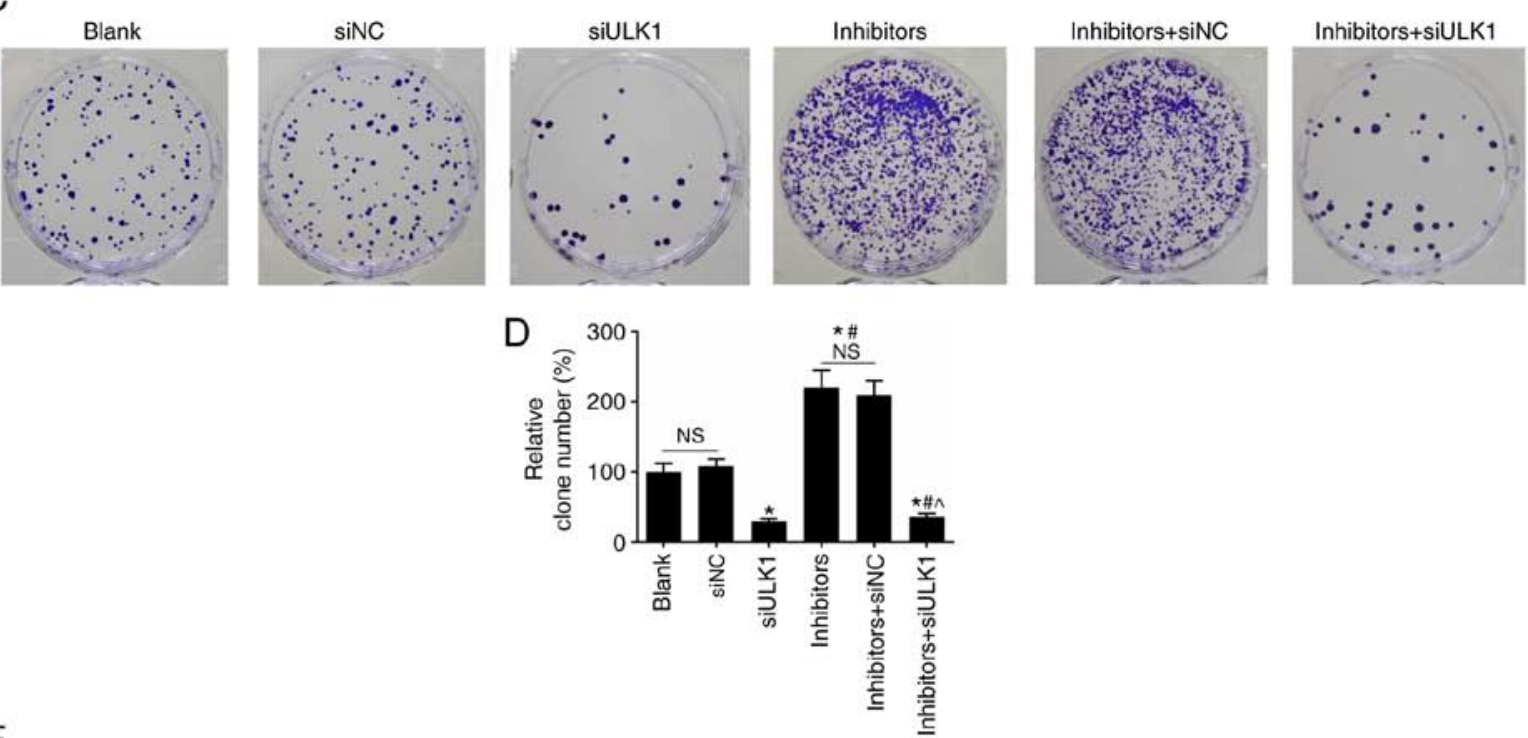

E
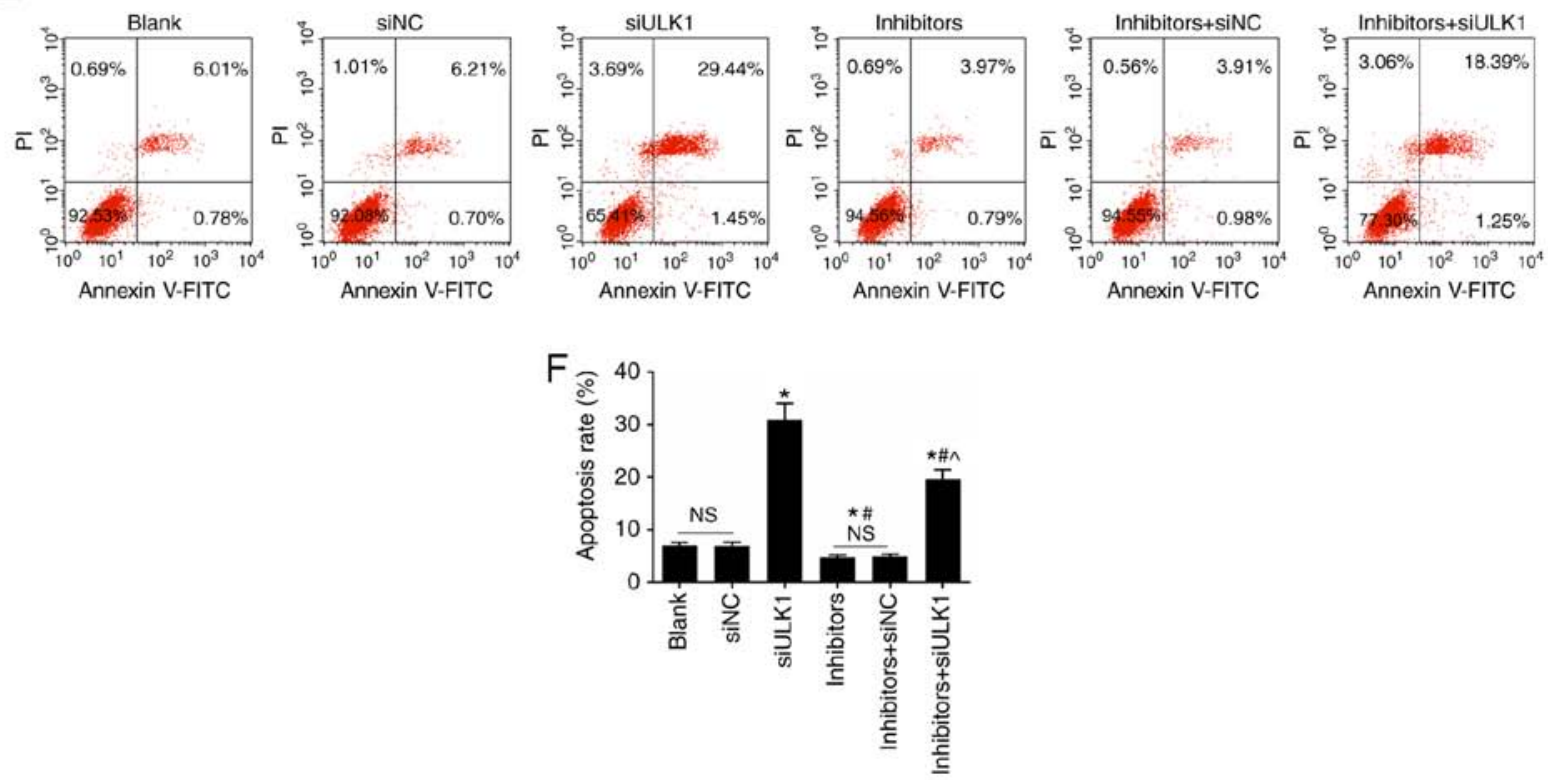

Figure 4. siULK1 inhibits CHON-001 cell migration and proliferation. (A and B) Scratch wound assay indicated that siULK1 inhibited CHON-001 cell migration. Scale bar, $200 \mu \mathrm{m}$. (C and D) Colony formation assay revealed that siULK1 had a negative effect on CHON-001 cell proliferation. (E and F) Flow cytometric assay indicated that siULK1 promoted apoptosis. The values were presented as the mean \pm standard deviation; data were analyzed by one way ANOVA. ${ }^{*} \mathrm{P}<0.05$ vs. Blank group, ${ }^{\wedge} \mathrm{P}<0.05$, vs. inhibitor + siNC group and ${ }^{\#} \mathrm{P}<0.05$ vs. siULK1 group. FITC, fluorescein isothiocyanate; miR, microRNA; NC, negative control; PI, propidium iodide; si, small interfering RNA; ULK1, Unc-51-like kinase 1. 

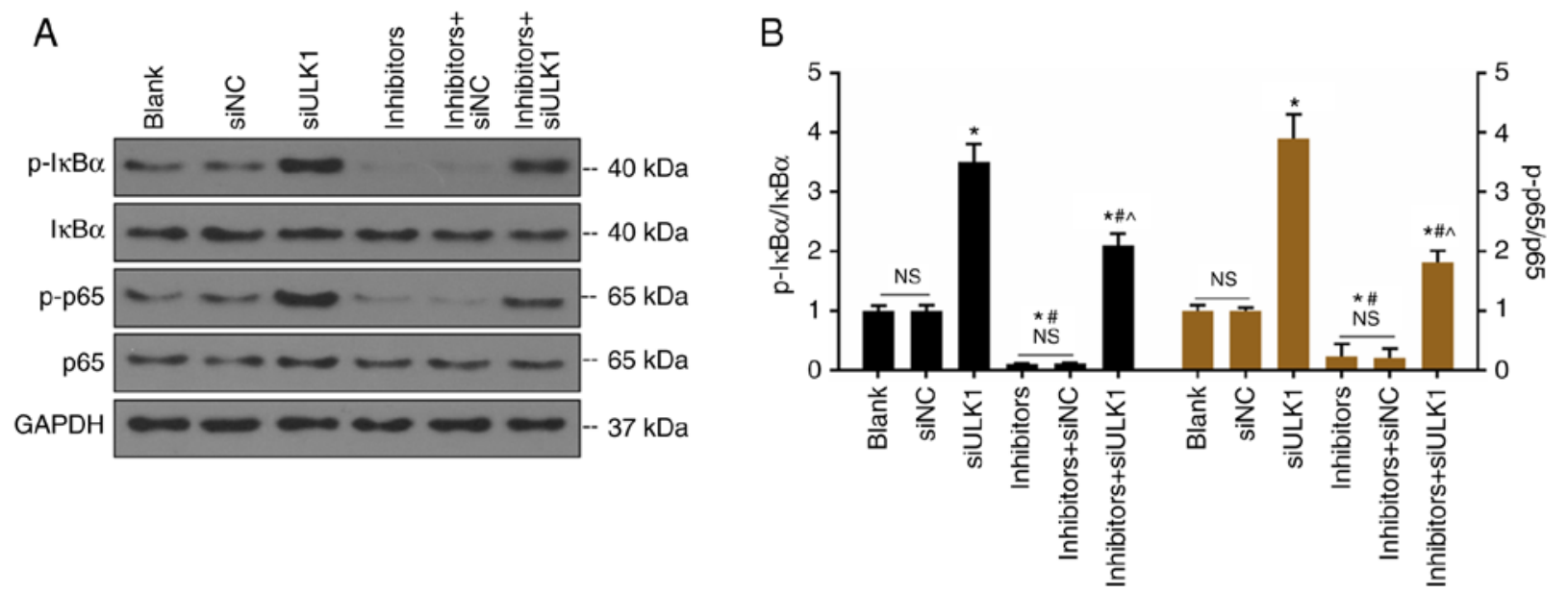

Figure 5. Effects of siULK1 and miR-302d-3p inhibitor on the expression of p-IкB $\alpha, \mathrm{I} \kappa \mathrm{B} \alpha, \mathrm{p}-\mathrm{p} 65$ and p65 in CHON-001 cells. (A and B) Western blotting for the detection of $\mathrm{p}-\mathrm{I} \kappa \mathrm{B} \alpha, \mathrm{I \kappa} \mathrm{B} \alpha, \mathrm{p}-\mathrm{p} 65$ and $\mathrm{p} 65$ protein expression after transfection with siULK1, miR-302d-3p inhibitor alone or in combination for $48 \mathrm{~h}$. The values were presented as the mean \pm standard deviation; data were analyzed by one way ANOVA. ${ }^{*} \mathrm{P}<0.05$ vs. Blank group; ${ }^{\wedge} \mathrm{P}<0.05$ vs. inhibitor + siNC group and " $\mathrm{P}<0.05$ vs. siULK1 group. miR, microRNA; NC, negative control; p, phosphorylated; si, small interfering RNA; ULK1, Unc-51-like kinase 1.

\section{Results}

Expression of $\mathrm{miR}-302 d-3 p$ and ULK1 in patient samples, and the effects of miR-302d-3p inhibitor or mimics on CHON-001 cell proliferation. Cartilaginous tissue of patients with OA had significantly higher miR-302d-3p expression levels compared with in normal cartilaginous tissue (Fig. 1A). Additionally, significantly lower expression levels of ULK1 were detected in the cartilaginous tissue of patients with OA (Fig. 1B-D). Transfection of cells with miR-302d-3p mimics resulted in a significant increase in miR-302d-3p expression (Fig. 1E) and significantly decreased $\mathrm{CHON}-001$ cell proliferation compared with in the blank control (Fig. 1F). On the contrary, miR-302d-3p inhibitor decreased miR-302d-3p expression and promoted CHON-001 cell proliferation compared with cells transfected with miR-302d-3p mimics (Fig. 1F).

Effects of miR-302d-3p inhibitor and mimics on CHON-001 cell migration, colony number and apoptosis. miR-302d-3p inhibitor significantly reduced the scratch distance and promoted $\mathrm{CHON}-001$ cell migration compared with the nontransfected control and miR-302d-3p mimics groups (Fig. 2A-B). The miR-302d-3p mimics group had long scratch distance and lower ability of migration in CHON-001 cells (Fig. 2A and B). Cells transfected with miR-302d-3p inhibitor exhibited a significant increase in colony number than the miR-302d-3p mimics group, which suggested that miR-302d-3p downregulation promoted the proliferation of CHON-001 cells (Fig. 2C and D). miR-302d-3p mimics significantly promoted apoptosis compared with nontransfected cells, but was suppressed in response to miR-302d-3p inhibitor transfection (Fig. 2E and F).

siULK1 suppresses CHON-001 cellproliferation. TargetScan7.2 was used to predict target genes of ULK1, which revealed miR-302d-3p as a potential target (Fig. 3A). This segment of the ULK1-3'UTR sequence (AGCACUU) is complementary to the miR-302d-3p wt sequence (UCGUGAA). The results of the dual luciferase report assay showed that miR-302d-3p mimics significantly decreased the luciferase activity of psi-CHECK-2 compared with the blank group, which supported that miR-302d-3p could bind to the 3'-UTR of ULK1 (Fig. 3B). Furthermore, siULK1 significantly inhibited ULK1 expression, while miR-302d-3p inhibitor increased ULK1 expression (Fig. 3C). siULK1 significantly inhibited the proliferation of CHON-001 cells compared with the inhibitor and siNC group, and miR-302d-3p inhibitor induced $\mathrm{CHON}-001$ cell proliferation (Fig. 3D).

siULK1 suppresses CHON-001 cell migration and colony number. The siULK1 group and miR-302d-3p inhibitor + siULK1 group had longer scratch distances compared that other groups, which suggested that siULK1 inhibited CHON-001 cell migration (Fig. 4A and B). Compared with the blank group, siULK1 significantly decreased CHON-001 cell colony number, whereas this was promoted by miR-302d-3p inhibitor, indicating that siULK1 reduced cell proliferation (Fig. 4C and D). In addition, siULK1 significantly promoted cell apoptosis compared with the nontransfected cell group (Fig. 4E and F).

Effects of miR-302d-3p inhibitor and siULK1 on the expres-

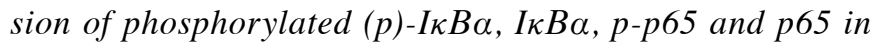
CHON-001 cells. siULK1 significantly promoted the protein expression of $\mathrm{p}-\mathrm{I} \kappa \mathrm{B} \alpha$ and $\mathrm{p}-\mathrm{p} 65$ compared with nontransfected cells, which supported that ULK1 knockdown may be associated with the activation of $\mathrm{I} \kappa \mathrm{B} \alpha$ and p65 in CHON-001 cells (Fig. 5A and B). miR-302d-3p inhibitor significantly suppressed the expression levels of $\mathrm{p}$-I $\mathrm{B} \mathrm{B} \alpha$ and $\mathrm{p}$-p65 compared with the nontransfected cell group, which indicated that miR-302d-3p inhibitor suppressed the activation of $\mathrm{I} \kappa \mathrm{B} \alpha$ and $\mathrm{p} 65$ in CHON-001 cells (Fig. 5A and B).

\section{Discussion}

ULK1 is serine/threonine protein kinase (STK) and has five ULK1 homologs, including ULK1, ULK2, ULK3, ULK4 and STK36; ULK1 and ULK2 have been reported to be involved in 
the mechanism of autophagy $(23,24)$. A recent report showed that activation of ULK4 inhibited autophagy and inflammatory responses (25). In our study, the cartilaginous tissue of OA patients had lower ULK1 expression levels, but miR-302d-3p was upregulated. Articular cartilage supports joint lubrication, and chondrocytes maintain the balance between the degradation and synthesis of the extracellular matrix (26). Musumeci et al supported that chondrocyte apoptosis was positively associated with cartilage destruction in patients with OA (27). Therefore, we explored the effects of miR-302d-3p inhibitor on chondrocyte proliferation and apoptosis in vitro. The results indicated that miR-302d-3p inhibitor inhibited the apoptosis and promoted the proliferation of chondrocytes. On the contrary, miR-302d-3p mimics promoted apoptosis and inhibited proliferation.

Cell migration serves a critical role in biological processes, including immune responses, tissue regeneration, wound healing and cancer metastasis (28). A scratch-wound assay is one simple method to measure cell migration by means of cellular movement (29). Chondrocyte migration is required for the repair of cartilage (30). Cartilage injury leads to the development of degenerative joint diseases such as OA (31). miR-302d-3p inhibitor promoted chondrocyte migration, but was inhibited in response to $\mathrm{miR}-302 \mathrm{~d}-3 \mathrm{p}$ mimics.

We used TargetScan7.2 to predict the target gene of miR-302d-3p, and a dual luciferase reporter assay was conducted. Our results revealed that ULK1 was one target gene of miR-302d-3p; RT-qPCR and western blotting verified that miR-302d-3p inhibitor increased ULK1 expression and siULK1 decreased ULK1 expression. Furthermore, siULK1 inhibited the proliferation and migration of chondrocytes, and miR-302d-3p inhibitor promoted chondrocyte proliferation.

$\mathrm{p} 65 / \mathrm{RelA}$ is a subunit of nuclear factor (NF)- $\kappa \mathrm{B}$ and is regarded as an important member of the NF- $\kappa \mathrm{B}$ pathway (32). IL-1 and TNF- $\alpha$ are well regarded as pro-inflammatory factors and are promptly released in injured tissues (33). A study has suggested that inflammatory factors, such as TNF- $\alpha$ and chemokines, can be induced by $N F-\kappa B$ activation in chondrocytes and synovial cells (34). NF- $\kappa \mathrm{B}$ is activated by multifarious stimulation, including Toll-like receptors, growth factor receptors, oxidation and genotoxic stress (35). IкB binds $\mathrm{NF}-\kappa \mathrm{B}$ dimers in the cytoplasm, preventing the $\mathrm{NF}-\kappa \mathrm{B}$ proteins from translocating to the nucleus to regulate gene expression; the I $\kappa \mathrm{B}$ proteins include: $\mathrm{I} \kappa \mathrm{B} \alpha, \mathrm{I} \kappa \mathrm{B} \beta$ and $\mathrm{I} \kappa \mathrm{B} \varepsilon(36)$. Therefore, the induction of $N F-\kappa B$ may depend on the phosphorylation of I $\mathrm{Bs}$ (37). siULK1 promoted the phosphorylation of $\mathrm{I} \kappa \mathrm{B} \alpha$ and p65, while miR-302d-3p inhibitor suppressed phosphorylation of $\mathrm{I} \kappa \mathrm{B} \alpha$ and p65 in chondrocytes, which indicated that miR-302d-3p inhibitor decreased chondrocyte inflammation.

In conclusion, we reported upregulated miR-302d-3p and decreased ULK1 mRNA expression levels in the cartilaginous tissue of OA patients. Additionally, inhibition of miR-302d-3p promoted the proliferation and migration, and inhibited the apoptosis of chondrocytes, suppressing inflammation. This may be due to the upregulated expression of ULK1. Inhibition of ULK1 had adverse effects compared with inhibition of miR-302d-3p in chondrocytes. Thus, downregulation of miR-302d-3p or upregulation of ULK1 may be considered as potential therapeutic strategies for preventing and treating $\mathrm{OA}$; however, further investigation using gene knock-out models and miR-302d-3p inhibitor treatment in vivo is required.

\section{Acknowledgements}

Not applicable.

\section{Funding}

No funding was received.

\section{Availability of data and materials}

The analyzed datasets generated during the study are available from the corresponding author on reasonable request.

\section{Authors' contributions}

Substantial contributions to conception and design: SW, YZhe and $\mathrm{ZH}$; data acquisition, data analysis and interpretation: ZW, YZha and LW; drafting the article or critically revising it for important intellectual content: LW, SW, YZhe. Agreement to be accountable for all aspects of the work in ensuring that questions related to the accuracy or integrity of the work are appropriately investigated and resolved: YZhe, YZha and $\mathrm{ZH}$. All authors approved the final version to be published.

\section{Ethics approval and consent to participate}

The present study was approved by the ethics committees and health authorities of The Second Affiliated Hospital of Henan University of Traditional Chinese Medicine (approval. no. R201703050089). All procedures performed in studies involving human participants were conducted in accordance with the ethical standards of the institutional and/or national research committee, and with the 1964 Helsinki declaration and its later amendments or comparable ethical standards. Written informed consent was obtained from all patients.

\section{Patient consent for publication}

Not applicable.

\section{Competing interests}

The authors declare no conflicts of interest.

\section{References}

1. Salaffi F, Carotti M, Stancati A and Grassi W: Health-related quality of life in older adults with symptomatic hip and knee osteoarthritis: A comparison with matched healthy controls. Aging Clin Exp Res 17: 255-263, 2005.

2. Brown TD, Johnston RC, Saltzman CL, Marsh JL and Buckwalter JA: Posttraumatic osteoarthritis: A first estimate of incidence, prevalence, and burden of disease. J Orthop Trauma 20: 739-744, 2006.

3. Muthuri SG, McWilliams DF, Doherty M and Zhang W: History of knee injuries and knee osteoarthritis: A meta-analysis of observational studies. Osteoarthritis Cartilage 19: 1286-1293, 2011.

4. Thomas AC, Hubbard-Turner T, Wikstrom EA and PalmieriSmith RM: Epidemiology of posttraumatic osteoarthritis. J Athl Train 52: 491-496, 2017

5. Spector TD and MacGregor AJ: Risk factors for osteoarthritis: Genetics. Osteoarthritis Cartilage 12 (Suppl A): S39-S44, 2004. 
6. Litwic A, Edwards MH, Dennison EM and Cooper C: Epidemiology and burden of osteoarthritis. Br Med Bull 105 185-199, 2013.

7. Woolf AD: Driving musculoskeletal health for Europe: EUMUSC. NET. Reumatismo 63: 1-4, 2011

8. Felson DT: Developments in the clinical understanding of osteoarthritis. Arthritis Res Ther 11: 203, 2009.

9. Pereira D, Ramos E and Branco J: Osteoarthritis. Acta Med Port 28: 99-106, 2015.

10. Shen J, Abu-Amer Y, O'Keefe RJ and McAlinden A: Inflammation and epigenetic regulation in osteoarthritis. Connect Tissue Res 58: 49-63, 2017

11. Su LC, Huang AF, Jia H, Liu $\mathrm{Y}$ and $\mathrm{Xu}$ WD: Role of microRNA-155 in rheumatoid arthritis. Int J Rheum Dis 20 $1631-1637,2017$

12. Acunzo M and Croce CM: MicroRNA in cancer and Cachexia-A mini-review. J Infect Dis 212 (Suppl 1): S74-S77, 2015.

13. Jiangpan P, Qingsheng M, Zhiwen Y and Tao Z: Emerging role of microRNA in neuropathic pain. Curr Drug Metab 17: 336-344, 2016.

14. Gao Z, Zhu X and Dou Y: The miR-302/367 cluster: A comprehensive update on its evolution and functions. Open Biol 5: $150138,2015$.

15. Wang Y, Baskerville S, Shenoy A, Babiarz JE, Baehner L and Blelloch R: Embryonic stem cell-specific microRNAs regulate the G1-S transition and promote rapid proliferation. Nat Genet 40: 1478-1483, 2008.

16. Xiao L, Jiang L, Hu Q and Li Y: MiR-302e attenuates allergic inflammation in vitro model by targeting RelA. Biosci Rep 38 BSR20180025, 2018.

17. Ma T, Liu X, Cen Z, Xin C, Guo M, Zou C, Song W, Xie R, Wang K, Zhou H, et al: MicroRNA-302b negatively regulates IL-1beta production in response to MSU crystals by targeting IRAK4 and EphA2. Arthritis Res Ther 20: 34, 2018.

18. Altman R, Asch E, Bloch D, Bole G, Borenstein D, Brandt K, Christy W, Cooke TD, Greenwald R and Hochberg M: Development of criteria for the classification and reporting of osteoarthritis. Classification of osteoarthritis of the knee. Diagnostic and Therapeutic Criteria Committee of the American Rheumatism Association. Arthritis Rheum 29: 1039-1049, 1986

19. Livak KJ and Schmittgen TD: Analysis of relative gene expression data using real-time quantitative PCR and the 2(-Delta Delta C(T)) method. Methods 25: 402-408, 2001.

20. Lim HD, Kim YS, Ko SH, Yoon IJ, Cho SG, Chun YH, Choi BJ and Kim EC: Cytoprotective and anti-inflammatory effects of melatonin in hydrogen peroxide-stimulated $\mathrm{CHON}-001$ human chondrocyte cell line and rabbit model of osteoarthritis via the SIRT1 pathway. J Pineal Res 53: 225-237, 2012.

21. Hu W, Zhang W, Li F, Guo F and Chen A: miR-139 is up-regulated in osteoarthritis and inhibits chondrocyte proliferation and migration possibly via suppressing EIF4G2 and IGF1R. Biochem Biophys Res Commun 474: 296-302, 2016.
22. Justus CR, Leffler N, Ruiz-Echevarria M and Yang LV: In vitro cell migration and invasion assays. J Vis Exp 2014.

23. Zachari $\mathrm{M}$ and Ganley IG: The mammalian ULK1 complex and autophagy initiation. Essays Biochem 61: 585-596, 2017.

24. Lin MG and Hurley JH: Structure and function of the ULK1 complex in autophagy. Curr Opin Cell Biol 39: 61-68, 2016.

25. He Y, She H, Zhang T, Xu H, Cheng L, Yepes M, Zhao Y and Mao Z: p38 MAPK inhibits autophagy and promotes microglial inflammatory responses by phosphorylating ULK1. J Cell Biol 217: 315-328, 2018

26. Musumeci G, Castrogiovanni P, Trovato FM, Weinberg AM, Al-Wasiyah MK, Alqahtani MH and Mobasheri A: Biomarkers of chondrocyte apoptosis and autophagy in osteoarthritis. Int J Mol Sci 16: 20560-20575, 2015.

27. Musumeci G, Aiello FC, Szychlinska MA, Di Rosa M, Castrogiovanni P and Mobasheri A: Osteoarthritis in the XXIst century: Risk factors and behaviours that influence disease onset and progression. Int J Mol Sci 16: 6093-6112, 2015.

28. Park JS, Rhau B, Hermann A, McNally KA, Zhou C, Gong D, Weiner OD, Conklin BR, Onuffer J and Lim WA: Synthetic control of mammalian-cell motility by engineering chemotaxis to an orthogonal bioinert chemical signal. Proc Natl Acad Sci USA 111: 5896-5901, 2014.

29. Cory G: Scratch-wound assay. Methods Mol Biol 769: 25-30, 2011.

30. Onuora S: Regenerative medicine. PBMCs stimulate chondrocyte migration and cartilage repair. Nat Rev Rheumatol 11: 563, 2015.

31. Jiang $Y$ and Tuan RS: Origin and function of cartilage stem/progenitor cells in osteoarthritis. Nat Rev Rheumatol 11: 206-212, 2015.

32. Chen S, Jiang S, Zheng W, Tu B, Liu S, Ruan H and Fan C: RelA/p65 inhibition prevents tendon adhesion by modulating inflammation, cell proliferation, and apoptosis. Cell Death Dis 8: e2710, 2017.

33. Lawrence T: The nuclear factor NF-kappaB pathway in inflammation. Cold Spring Harb Perspect Biol 1: a001651, 2009.

34. Malfait AM: Osteoarthritis year in review 2015: Biology. Osteoarthritis Cartilage 24: 21-26, 2016.

35. Christian F, Smith EL and Carmody RJ: The regulation of NF-kappaB subunits by phosphorylation. Cells 5: E12, 2016.

36. Fernandez G, Zaikos TD, Khan SZ, Jacobi AM, Behlke MA and Zeichner SL: Targeting IkappaB proteins for HIV latency activation: The role of individual IkappaB and NF-kappaB proteins. J Virol 87: 3966-3978, 2013.

37. Hinz $M$ and Scheidereit C: The IkappaB kinase complex in NF-kappaB regulation and beyond. EMBO Rep 15: 46-61, 2014.

This work is licensed under a Creative Commons Attribution-NonCommercial-NoDerivatives 4.0 International (CC BY-NC-ND 4.0) License. 